\title{
Analysis of the distribution of ingested bacteria in nanoflagellates and estimation of grazing rates with flow cytometry
}

\author{
Delma Bratvold ${ }^{1, *}$, Friedrich Srienc ${ }^{2}$, Stephan R. Taub ${ }^{1}$ \\ ${ }^{1}$ Biology Department, George Mason University, Fairfax, Virginia 22030-4444, USA \\ ${ }^{2}$ Department of Chemical Engineering and Materials Science, Institute for Advanced Studies of Biological Process Technology, \\ University of Minnesota, Minneapolis, Minnesota 55455, USA
}

\begin{abstract}
The distribution of ingested bacteria in nanoflagellates was assessed to suggest whether or not there are subgroups of grazers with different grazing rates. Several discrete random distributions were compared to the distribution of ingested fluorescently labeled bacteria (FLB) in cultures of Rhynchomonas nasuta and Paraphysomonas vestita. Sample distributions typically fit both the Poisson with extra zeros (Poisson EZ, tested as a truncated Poisson) and negative binomial, but only occasionally fit a Poisson. Both the Poisson EZ and the negative binomial distributions suggest a heterogeneous population composed of subgroups of flagellates with different grazing rates. Although these models provide acceptable mathematical descriptions, their specific biological implications with regard to the number of flagellate subgroups remain to be proven. Based on fit of the distribution of ingested prey to a Poisson EZ, a rapid cytometric method for estimation of grazing rates on FLB is presented. The method uses changes in the probability of grazers not ingesting FLB during short incubations (ca $15 \mathrm{~min}$ ) to estimate the Poisson parameter and the fraction of extra zeros, from which the average grazing rate is calculated. Grazing rates determined by microscopy and by this cytometry method were similar. Frequency distributions of cytometric histograms of fluorescent microspheres in grazers suggest that both the Poisson EZ and negative binomial models are simplifications of a more complex distribution of grazing rates.
\end{abstract}

KEY WORDS: Flagellate - Grazing rate - Truncated Poisson - Negative binomial - Flow cytometry

\section{INTRODUCTION}

The presence of bacterivorous flagellates in aquatic systems has been reported to cause increases in both primary and secondary productivity (Andersson et al. 1986, Bjørnsen et al. 1988, Roman et al. 1988, Sherr et al. 1988a, Ferrier \& Rassoulzadegan 1991, Rothhaupt 1992, Ferrier-Pagès \& Rassoulzadegan 1994). Productivity increases may result from enhanced nutrient recycling associated with bacterivory (Stout 1977, Sieburth \& Davis 1982, Goldman, et al. 1985, Caron \& Goldman 1988, Sherr et al. 1988a, Bloem et al. 1989,

-Present address: South Carolina Department of Natural Resources, Waddell Mariculture Center, PO Box 809. Bluffton, South Carolina 29910, USA. E-mail: bratvold@musc.edu
Selmer et al. 1993, Hutchins \& Bruland 1994). These concepts have stimulated interest in measurement of flagellate grazing on bacteria.

Estimates of grazing rates on bacteria are typically based on measurements of mean bacteria consumed per grazer. Understanding the distribution of prey in grazers (i.e. the number of flagellates containing 0,1 , 2 , etc., bacteria) may assist in the selection of adequate sample sizes and method development for grazing rate analysis. Taking into account the random chance of a flagellate encountering a bacterium, the distribution of grazing rates can also suggest biological conditions such as whether or not the entire bacterivore population is actively feeding, and whether or not active feeders have more than 1 grazing rate. Heterogeneous grazing rates for a single population of grazers may 
result from factors such as genetic and life cycle variability, or heterogeneous spacial distribution of grazers with respect to prey. In addition to these variables, heterogeneous grazing rates in natural communities may reflect multiple species-dependent grazing rates.

There are 3 common methods for estimating flagellate bacterivory rates: (1) via bacterial growth with and without grazers (e.g. Fenchel 1982, Davis \& Sieburth 1984), (2) via grazer uptake of radiolabeled bacteria (e.g. Hollisbaugh et al. 1980, Nygaard \& Hessen 1990), and (3) via grazer uptake of fluorescently labeled prey (e.g. McManus \& Fuhrman 1986, Sherr et al. 1987, Landry et al. 1991). Microscopic analysis of ingested fluorescently labeled prey, such as used by Hondeveld et al. (1987), Sherr et al. (1987, 1989), Sieracki et al. (1987), Nygaard et al. (1988), and Gonzalez et al. (1990) is the only method currently available that can estimate the distribution of prey ingested by individual grazers. Studies using this method generally have sample sizes of 100 to 300 flagellates. Labeled prey as a percentage of total prey is kept as low as possible to avoid increasing grazing rates due to significant increases in prey density (McManus \& Okubo 1991). Typically, only 5 to $30 \%$ of the prey is labeled, yielding a mean of labeled prey per flagellate that is less than 1 , and a significant number of feeding flagellates that may not have encountered labeled prey within incubation times that are less than digestion times. This can result in the absence of labeled prey in an actively feeding grazer. Furthermore, because of the low mean labeled prey per grazer, large sample sizes are required for good estimation of grazing rates.

After incubation of grazers with fluorescently labeled bacteria (FLB), the percentage of flagellates containing labeled prey has been reported to vary from 2 to $97 \%$ (Hondeveld et al. 1987, Sieracki et al. 1987, Nygaard et al. 1988, Sherr et al. 1989), depending upon factors such as the proportion and type of labeled prey, incubation time and conditions, grazer type, and fixation methods. The large number of flagellates with no ingested FLB led Hondeveld et al. (1987) to calculate grazing rates via 2 methods: (1) average FLB ingested by the entire flagellate sample, and (2) average FLB ingested by flagellates containing at least 1 FLB. The latter of these methods does not account for the probability of a feeding flagellate not encountering an FLB, and therefore overestimates grazing rates of the feeding population. If flagellate ingestion of FLB is due solely to the chances of a flagellate encountering an FLB, and flagellates and FLB are homogeneously distributed, then grazing rates should be homogeneous and the distribution of FLB in flagellates should fit a Poisson. McManus \& Okubo (1991) found the distribution of FLB in flagellate grazers did not fit a Poisson, which suggests the chances of a grazer ingesting an
FLB are not the same for all flagellates in a sample (i.e. the rates are heterogeneous). In contrast, Gonzalez (1999) used indirect methods to suggest that the distribution of ingested FLB fits a Poisson. In the present study, several discrete random models, with different biological implications with regard to the homogeneity and heterogeneity of grazing rates, are compared to sample distributions of ingested bacteria per grazer. One of the accepted model distributions, the Poisson with extra zeros (Poisson EZ), is then used to develop a cytometric method for measuring flagellate grazing rates.

Standard methods for estimating nanoflagellate grazing rates via microscopic analysis of labeled prey uptake are very tedious, requiring continual microscope refocusing and slide advancement, and thus are subject to variable degrees of operator error in data collection. Cytometry offers the possibility of rapid assessment (e.g. 2 to $3 \mathrm{~min}$ ) of very large sample sizes (e.g. 20000 cells). The benefit of large sample sizes may be particularly important for the low-mean samples often seen in grazing experiments. Cytometric methods have been developed for measurement of ciliate grazing rates on fluorescently labeled microspheres (Lavin et al. 1990. Fredrickson et al. 1992). However, because flagellates typically have lower grazing rates on microspheres than on labeled bacteria (Pace \& Bailiff 1987, Sherr et al. 1987, Nygaard et al. 1988) estimation of flagellate grazing rates using FLB is often preferable. This study presents a cytometric method for estimation of flagellate grazing rates on FLB.

\section{MATERIAL AND METHODS}

Grazing experiments. Clonal flagellate cultures were established from 2 Chesapeake Bay tributaries as described in Bratvold (1995). Cultures were maintained in Cerophyll medium (Nerad \& Daggett 1992) with salinities similar to, and mixed bacterial assemblages from, the site of their collection (i.e. a salinity of 15 for Rhynchomonas nasuta, and freshwater for Paraphysomonas vestita). For grazing measurements of $R$. nasuta, FLB incubations were conducted when the culture was near maximum flagellate abundances for this system (i.e. around $1 \times 10^{6}$ flagellates $\mathrm{ml}^{-1}$ ), in $50 \mathrm{ml}$ of culture according to the methods of Sherr et al. (1987). FLB comprised 4 to $12 \%$ of the total bacterial abundance, which was typically around $5 \times 10^{8}$ bacteria $\mathrm{ml}^{-1}$. Grazing was stopped after 10,15 , or 20 min incubation with the addition of $1.25 \%$ glutaraldehyde, final volume.

In later grazing experiments with exponential phase Paraphysomonas vestita (approximately $1 \mathrm{~d}$ old flagellate cultures), FLB were prepared from $2 \mathrm{~d}$ old bacterial cultures incubated $2 \mathrm{~h}$ in $0.02 \mathrm{M}$ carbonate buffer, 
pH 9.3, $25^{\circ} \mathrm{C}$, with approximately $0.2 \mathrm{mg}$ 5- $(4,6-$ dichlorotriazin-2-yl) aminofluorescein (DTAF) $\mathrm{ml}^{-1}$. The increased binding affinity of DTAF at high $\mathrm{pH}$ precluded the need for high temperature incubations as in Sherr et al. (1987). Labeled bacteria were washed and resuspended in $0.01 \mathrm{M}$ phosphate buffer ( $\mathrm{pH} 7.4$ ), as were $1.0 \mu \mathrm{m}$ diameter yellow-green fluorescent microspheres (Polysciences, Inc., Warrington, PA). Electron microscopic analysis of similarly prepared FLB suggests that structural integrity of the bacterial outer paracrystalline protein surface layer (S-layer) is maintained with this high $\mathrm{pH}$ treatment (S. Koval pers comm.). On the day of grazing experiments, exponential phase $P$. vestita cultures were passed through a $10 \mu \mathrm{m}$ pore size filter, and labeled prey was added to the filtered cultures as 6 to $22 \%$ of the total prey population. For microscopic analysis of $P$. vestita grazing rates, $2 \mathrm{ml}$ aliquots were taken after 10 or $12 \mathrm{~min}$ incubation with FLB, added directly to tubes containing $40 \mu$ of saturated $\mathrm{NiCl}_{2}$ (in distilled water), and placed in an ice bath. Nickel preservation avoided $P$. vestita fluorescence due to aldehyde preservation, which is a particular issue for this species. Samples were microscopically examined on shallow hanging-drop slides within $12 \mathrm{~h}$ of sample collection. Slide illumination was alternated between bright field for identification of flagellates, and fluorescence for examination of ingested FLB.

For cytometric analysis of grazing rates, sample aliquots were taken immediately after labeled prey addition, and every $3 \mathrm{~min}$ for $15 \mathrm{~min}$. Upon aliquot collection, grazing was stopped by a low temperature treatment, in which $2 \mathrm{ml}$ aliquots were added directly to tubes containing $2 \mathrm{ml}$ ice-cold, $0.2 \mu \mathrm{m}$ filtered, $0.01 \mathrm{M}$ phosphate buffer and placed in a saline ice bath $\left(0\right.$ to $\left.-2^{\circ} \mathrm{C}\right)$. Cold sample tubes were placed on the cytometer in a beaker of saline ice water immediately prior to analysis, which was completed within $10 \mathrm{~h}$ of sample collection.

For both Rhynchomonas nasuta and Paraphysomonas vestita studies, bacterial and flagellate abundances were determined from samples preserved with $1.25 \%$ final volume buffered glutaraldehyde. Refrigerated glutaraldehyde preserved samples were stained with 4,6-diamidino-2-phenyl-indol dihydrochloride (DAPI) (Porter \& Feig 1980) and examined with fluorescence microscopy for abundance measurements of unlabeled bacteria, FLB, and flagellates within 2 wk of sample collection.

Calculation of grazing rates from frequency distributions. Data were collected as frequency distributions of the number of flagellates containing $0,1,2$, etc., labeled prey. Four and 6 ingested FLB per flagellate were the maximum numbers (i.e. classes) that could be confidently enumerated for Rhynchomonas nasuta and
Paraphysomonas vestita, respectively. Data for these maximum classes were reported as greater than or equal to the enumerated FLB. Frequency distributions were collected from microscopic analysis of ingested FLB and from cytometric analysis of ingested fluorescent microspheres. Grazing rates were estimated by multiplying the mean number of labeled prey per grazer per minute by the ratio of total prey to labeled prey. For the flow cytometer data, histogram counts of grazer-associated microspheres collected immediately after FLB additions to grazers (i.e. a 'time-zero' blank) were subtracted from later histogram counts to adjust for enumeration of microspheres that were not ingested by flagellates.

Comparison of sample and model distributions. To find an appropriate model distribution of prey ingested by grazers, a 2-step process was employed. First, the frequency distribution of the largest sample of microscopically analyzed Rhynchomonas nasuta was compared to 4 different model distributions using the chisquare $\left(\chi^{2}\right)$ test for goodness of fit (Sokal \& Rohlf 1981), with $\alpha=0.05$ and the appropriate degrees of freedom for the hypothesized model. Classes with expected frequencies less than 1 were grouped with their neighboring class. However, model acceptance in a single sample may better explain natural variability in that particular sample than in a population (Bishop et al. 1975). Therefore, in a second step, the 2 model distributions that best fit the largest sample distribution were postulated as models for all other samples. For each postulated model, the probability value of the $\chi^{2}$ test statistic for each $R$. nasuta sample was placed in its appropriate percentile group (percentile group ranges are shown in Table 2). The frequency distribution of the percentile groups was then compared to the expected percentile frequencies with the $\chi^{2}$ test for goodness of fit.

The 4 mathematical models considered in this study are common, discrete random distributions: the binomial, Poisson, Poisson EZ, and negative binomial. The parameters or quantities needed to define a binomial distribution are $n$ and $p$. The random variable $x$ is the number of FLB in a given grazer. The probability of the random variable $x[$ written as $P(x)]$ is:

$$
P(x)=\frac{n ! p^{x}(1-p)^{n-x}}{x !(n-x) !} \quad \text { for } x=(0,1, \ldots, n)
$$

The distribution of ingested labeled bacteria per grazer can be thought of as binomial if there are $n$ possible food slots per grazer (determined by microscopic examination of the maximum number of ingested bacteria in a grazer), and the probability of a food slot containing a labeled bacterium is $p$, then there are $x$ labeled bacteria per flagellate. The mean of this distribution is $n p$; thus $p$ can be estimated from a sample as 
$\mathrm{m} / \mathrm{n}$, where $m$ is the sample mean. The fit of grazing data to a binomial suggests independence in the probability of a food slot being full.

The Poisson distribution requires a single parameter $\lambda$ to define the distribution. This parameter is equal to both the distribution mean and variance. The random variable of this distribution, $x$, is the number of FLB in a given grazer. The probability of $x$ is:

$$
P(x)=\frac{\lambda^{x} \mathrm{e}^{-\lambda}}{x !} \quad \text { for } x=(0,1, \ldots, n) \text { and } \lambda>0
$$

The Poisson parameter $\lambda$ is estimated as the mean number of FLB per grazer in the sample distribution at the sample incubation time. The fit of a sample to a Poisson suggests that the cause of different numbers of FLB in different flagcllates is solely due to the chance of a flagellate encountering an FLB. Thus, there are no differences in the grazing rates of individual flagellates (i.e. rates are homogeneous), assuming the prey is homogeneously distributed. However, it may be inappropriate to assume a single grazing rate based on the fit of a sample with a low mean to a Poisson. The law of small numbers states that low frequency events in a large population can be fit to a Poisson even when the probability of events varies among subgroups of the population (Gumbel 1968). Therefore, samples that have a low mean number of ingested FLB per grazer (e.g. <1) may have several different grazing rates and still appear to fit a Poisson. Examination of large sample sizes (e.g. $>1000$ ) increases the chances of being able to detect multiple grazing rates in these populations because it allows the tails of the distribution to be better defined, making it easier to detect poor fit to a Poisson.

If a sample frequency distribution has a zero class that is significantly larger than expected for a Poisson distribution, a Poisson EZ may be tested with a Poisson truncated at the one class (i.e. data for the zero class is not included). The parameter for the truncated Poisson is $\lambda_{T}$, and the mean of the truncated distribution is $m_{r}$. With denotation of an estimate by a prime ('), the initial $\lambda_{T^{\prime}}$ is $m_{T}$, and $\lambda_{T}$ ' is iteratively computed (El-Shaarawi et al. 1981) as:

$$
\lambda_{1}^{\prime}=m_{\uparrow}\left(1-e^{-\lambda p^{\prime}}\right)
$$

where the initial $\lambda_{T}{ }^{\prime}$ is $\lambda_{0}{ }^{\prime}$, and the next $\lambda_{T}{ }^{\prime}$ is $\lambda_{1}{ }^{\prime} \cdot \lambda_{1}{ }^{\prime}$ then replaces $\lambda_{0}{ }^{\prime}$ to obtain a better $\lambda_{\mathrm{T}}{ }^{\prime}$. This process is repeated until the difference between $\lambda_{0}{ }^{\prime}$ and $\lambda_{1}{ }^{\prime}$ becomes very small (e.g. $<0.0001)$. It should be noted that truncated Poisson parameter estimation methods yield an invalid estimate whenever the sample does not include an individual(s) in a class greater than 1 ; thus, a sufficiently large sample must be analyzed to include grazers that have consumed more than 1 labeled prey (Bratvold 1995). Given that for the Pois- son the probability that there is more than 1 ingested FLB in a grazer is $P_{(x>0)}=\left(1-e^{-\lambda}\right)$, the number of individuals in the Poisson $\left(N_{p}\right)$ can be estimated from $\lambda_{T}$ as:

$$
N_{\mathrm{p}}{ }^{\prime}=\frac{\sum_{x=1}^{\infty} f_{x}}{\left(1-\mathrm{e}^{-\lambda_{T}}\right)}
$$

where $f_{\mathrm{x}}$ is then number of flagellates in the sample that contain $x$ FLB. $N_{p}$ ' can then be subtracted from the sample size (including the zero class) to estimate the number of extra zeros, which, if the theoretical implications of the Poisson are true, would correspond to grazers that were not ingesting bacteria even if they encountered them during the incubation.

When both tails of the sample distribution have greater frequencies than expected for a Polsson distribution, a negative binomial model may better fit the sample. The negative binomial can be thought of as a series of many overlapping Poisson distributions (i.e. a compound Poisson), where each subgroup of flagellates has a grazing rate that can be fit to a Poisson. The negative binomial is, arguably, the most common type of compound Poisson. The fit of grazing samples to a negative binomial suggests the presence of multiple grazing rates. The negative binomial is defined by 2 parameters: $k$, and the arithmetic mean of the sample, $m$. The random variable $x$ is the number of labeled bacteria in a flagellate, and

$$
P(x)=\frac{(k+x-1) !\left[\frac{m}{(k+m)}\right]^{x}}{x !(k-1) !\left(1+\frac{m}{k}\right)^{k}} \text { for } x=(0,1, \ldots, n)
$$

An initial estimate of the parameter $k$ can be calculated as $k^{\prime}=m^{2} /\left(s^{2}-m\right)$, where $s^{2}$ is the sample variance (Bliss \& Fisher 1953). A final estimate of $k$ is determined by iterative calculations to approach $b=0$ when

$$
b=\sum_{x=0}^{n}\left(\frac{\sum_{x_{1}+1}^{n} f_{x}}{k^{\prime}+x}\right)-N \ln \left(\frac{1+m}{k^{\prime}}\right)
$$

where $N$ is the number of grazers in the sample and $x_{1}$ is the lowest number of FLB observed in a grazer (i.e. zero). With each iteration, $k^{\prime}$ is adjusted until the absolute value of $b$ is sufficiently small (i.e. $b<0.005$ in this study).

Flow cytometry analysis. Flow cytometry was conducted with an Or tho Cytofluorograf IIs flow cytometer (Ortho Diagnostic Systems, Westwood, MA), Data were acquired, displayed, and stored with an Ortho 2151 data acquisition and analysis system (Ortho Diagnostics Systems). Three signals were measured and stored for each individual cell: (1) fluorescence intensity, primarily from ingested labeled prey, (2) forward angle 
light scatter (FALS), which roughly correlates with grazer size, and (3) right angle light scatter (RALS), which roughly correlates with cell granularity. Signals were processed in area mode, i.e. the integral of emitted light over time. Fluorescence measurements used a Coherent Innova 90-5 argon ion laser (Coherent, Palo Alto, CA) for illumination at $488 \mathrm{~nm}$, with a light-stabilized power output of $100 \mathrm{~mW}$, and a 515 to $530 \mathrm{~nm}$ barrier filter (Ortho Diagnostics Systems).

Two-dimensional cytograms of FALS versus RALS (in which each point represents the FALS and RALS characteristics of a single cell) were used to adjust RALS and FALS gain settings for optimal separation of bacterial and flagellate populations. FALS versus fluorescence cytograms were used to quantify fluorescence incorporated into flagellates. Cytometric separation of flagellate and uningested bacterial populations was initially tested with monoflagellate cultures of Cafeteria sp., Jakoba libera, Paraphysomonas vestita, and $P$. butcherii. Cultures were passed through a $10 \mu \mathrm{m}$ pore size filter prior to cytometric analysis to remove large bacterial clumps. $P$. vestita and Cafeteria sp. were clearly separated from their resident bacterial populations based on FALS and RALS. A cytogram of $P$. vestita and bacterial populations is shown in Fig. 1; the population concentrated in the lower FALS and RALS channels represents the bacterial population, while the population concentrated in the higher channels represents the flagellate population. Cytographic overlap of bacterial and flagellate populations was greater in $J$. libera and $P$. butcherii cultures than in $P$. vestita and Cafeteria sp. cultures, but distinct bacterial and flagellate peaks were seen in FALS histograms of all flagellates tested.

Paraphysomonas vestita was used throughout the remainder of the cytometry portion of this study because of its rapid growth and good cytometric separa-

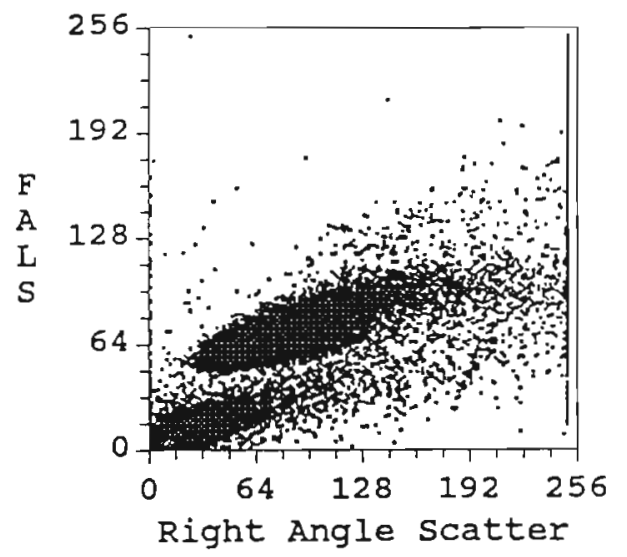

Fig. 1. Separation of bacterial and Paraphysomonas vestita populations. FALS: forward angle light scatter tion. In preliminary trials with different fixatives it was found that formaldehyde and glutaraldehyde fixation increased cell fluorescence and generally decreased cytometric separation of the grazer and prey communities. $\mathrm{NiCl}_{2}$ fixation had minimal effects on population separations but slow formation of a precipitate was a concern. Low temperature fixation had negligible effects on population separations and was used for the remainder of this study. This treatment caused cessation of flagellate movement without death (i.e. incubations of $P$. vestita and FLB at low temperature for up to $12 \mathrm{~h}$ did not show significant change in FLB-related flagellate fluorescence and the flagellates regained motility when allowed to return to room temperature).

Cytometric histograms of ingested FLB and fluorescent microspheres have different appearances. FALS versus fluorescence histograms of flagellates after incubation with microspheres show distinct peaks for grazers containing $1,2,3$, and 4 or more fluorescent microspheres (Fig. 2A). However, similar histograms of flagellates incubated with FLB do not show distinct fluorescence peaks corresponding to the number of labeled prey ingested per cell (Fig. 2B). This is a result of the relatively wide range of bacterial sizes and fluorescence intensities, which causes a general increase in flagellate fluorescence after incubation with FLB. The percentage of total cell counts in selected fluorescence channel groups are displayed over the brackets in Fig. 2. For microsphere uptake, fluorescence channel groups were selected based on channels assumed to correspond to $0,1,2,3$, and 4 or more ingested microspheres. For FLB uptake, fluorescence channel groups were selected based on channels corresponding to no FLB uptake, determined from grazer population fluorescence measured immediately after mixing with FLB, and channels corresponding to 1 or more ingested FLB. Typically 20000 flagellates were examined per sample at a rate of 300 to 500 cells s$^{-1}$. For samples incubated with fluorescent microspheres, at least 40000 flagellates were analyzed to obtain distinct peaks in the higher classes of ingested microspheres.

Calculation of grazing rates from probability of zero curves. This method can be used when samples fit a Poisson EZ distribution. It requires estimation of the fraction of flagellates that have no ingested FLB

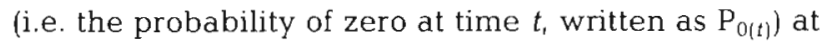
several time points throughout a short incubation with FLB. The data curve of grazers with no ingested FLB versus time is then compared to a theoretical curve that is repeatedly re-drawn with different estimates of the model parameters until the theoretical curve overlies the data curve. The parameter estimates used in the final theoretical curve are used to calculate overall grazing rates. Based on the Poisson probability of zero, $\mathrm{P}_{0}=\mathrm{e}^{-\lambda}$ (derived from Eq. 2), a curve of the Poisson EZ 


\section{A. Microsphere Uptake}

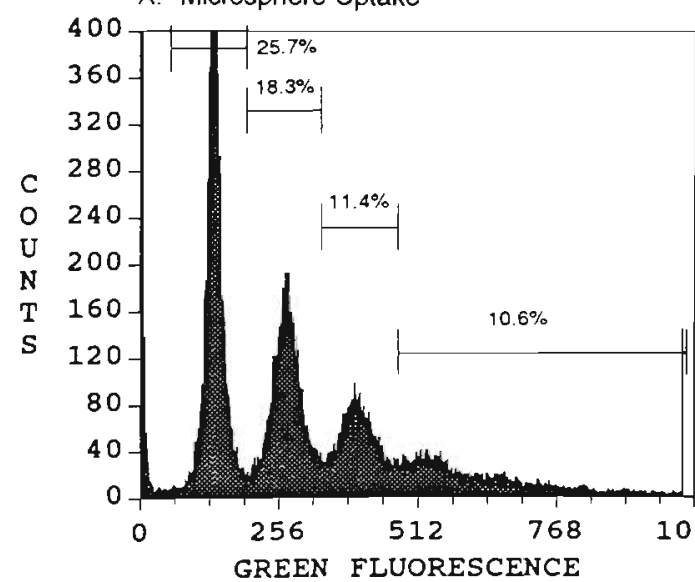

B. FLB Uptake

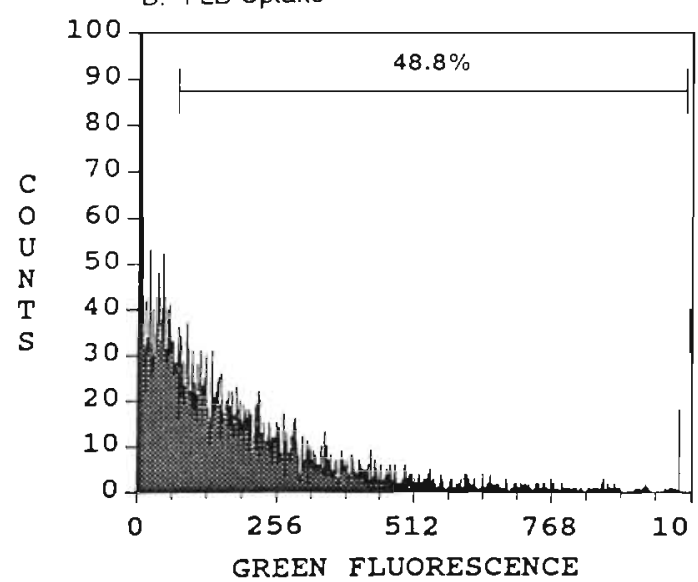

Fig. 2. Paraphysomonas vestita fluorescence after incubation with (A) $1 \mu \mathrm{m}$ fluorescent microspheres, and (B) FLB for $12 \mathrm{~min}$

probability of zero at time $t$ versus time can be constructed from:

$$
\mathrm{P}_{0(t)}=(1-z) \mathrm{e}^{-\lambda t}+z
$$

where $\mathrm{P}_{0(t)}$ is the probability of flagellates having no ingested labeled prey at time $t$, and $z$ is the fraction of extra zeros that do not fit in a Poisson distribution (i.e. in the case of sample fit to a Poisson, $z=0$ ). $\lambda$ is the Poisson parameter in units of FLB per grazer per base unit time (i.e. per 1 min rather than per incubation time as in Eq. 2). Theoretical curves were visually compared to sample $\mathrm{P}_{0(\ell)}$ curves with a minimum of 5 time points. Although not applied in this study, a least squares method or a Poisson regression could be used in place of visual fit.

An understanding of the behavior of theoretical $\mathrm{P}_{0(t)}$ curves assisted in the selection of appropriate model curves to approximate $P_{0,4)}$ data points. When the sample distribution of prey ingested per grazer has more zeros than expected in the Poisson distribution, with increasing incubation time, $\mathrm{P}_{0(t)}$ approaches the frac- tion of extra zeros rather than zero. Fig. 3 shows a series of theoretical $\mathrm{P}_{0(t)}$ versus time curves for Poissons with no extra zeros, and with 20 and $50 \%$ of the sample composed of extra zeros. Fig. 3A shows that for $\lambda \geq 0.5, z$ can be estimated from $\mathrm{P}_{0(t)}$ at $12 \mathrm{~min}$ and beyond. Fig. $3 B$ shows that when $\lambda \leq 0.1, \mathrm{P}_{0(t)}$ measured at $20 \mathrm{~min}$ is an overestimate of $z$. However, $\lambda$ can still be estimated by initially drawing a curve (Eq. 7) with the last measured $\mathrm{P}_{0(1)}$ as an estimate of $z$, and then redrawing the curve with $\lambda$ adjustments and progressively lower $z$ until a good fit is found. Fig. $3 \mathrm{C}$ shows that when $\lambda \leq 0.01$, the measured portion of the curve approaches a line. The same line can be described by both a Poisson parameter (with $z=0$ ) and a different set of Poisson EZ parameters, thus this method fails when $\lambda$ is very low.

Incubation times in excess of digestion time cannot be used because an asymptote greater than zero may be reached in the $P_{0(t)}$ curve due to either true

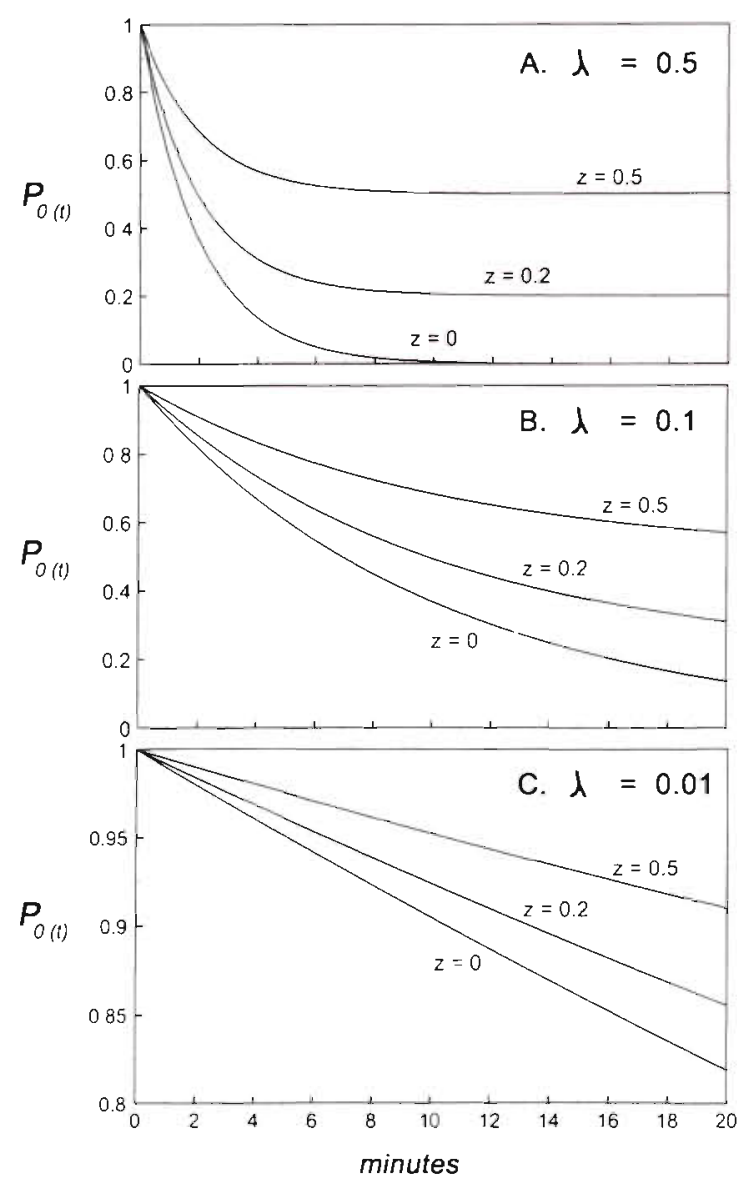

Fig. 3. Theoretical probability of zero $\left[\mathrm{P}_{0}\right.$. $]$ versus time curves for Poisson EZ (Poisson with extra zeros) distributions when the fraction of extra zeros $(z)=0,0.2$ and 0.5 of the entire population, and the Poisson parameter $(\lambda)=(A) 0.5$, (B) 0.1 . and $(\mathrm{C}) 0.01$ 


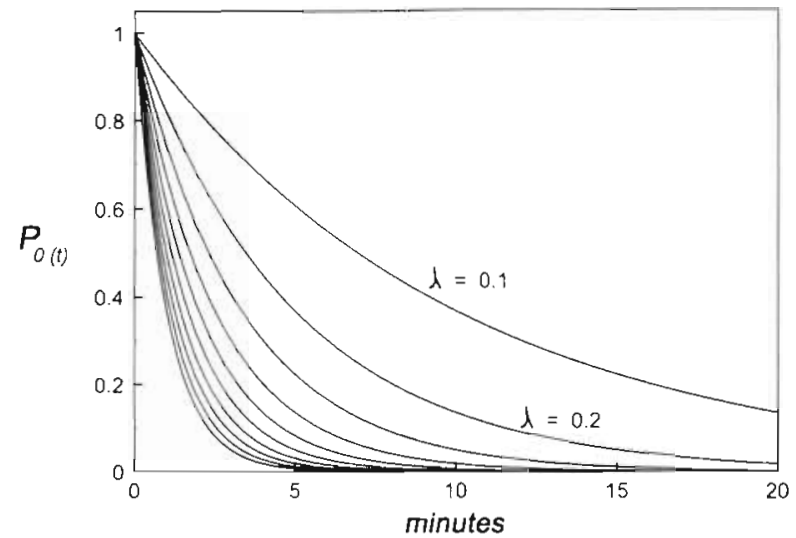

Fig. 4. Theoretical probability of zero $\left[\mathrm{P}_{\mathrm{D}(\mathrm{i})}\right]$ versus time curves for Poisson distributions with the Poisson parameter $(\lambda)=0.1$, $0.2,0.3, \ldots, 1.0$

fit to a Poisson EZ model, or digestion of FLB prior to capture of another labeled prey. Digestion time is dependent upon experimental conditions such as grazer and prey type (Sherr et al. 1983, Goldman \& Caron 1985, Fenchel 1987), and temperature (Sherr et al. 1988b), and has been reported to range from 10 min to $1 \mathrm{~h}$ (Sherr et al. 1988b, Gonzalez et al. 1990). Using a Poisson with no extra zeros, Fig. 4 shows that as $\lambda$ increases, theoretical $P_{0(t)}$ curves are closer together. Thus, smaller $\lambda$ can be estimated with more precision, until the curve approaches a line, as in Fig. 3C. Smaller $\lambda$ can be achieved with lower concentrations of labeled prey.

The Poisson parameter $\lambda$ should not be assumed to represent the grazing rate of a feeding subpopulation unless it is confirmed that the extra zeros in the Poisson EZ model correspond to non-feeders. Thus, grazing rates should be expressed for the entire flagellate population. After $z$ and $\lambda$ are estimated from data $P_{\text {o(t) }}$ curve fit to a theoretical curve, the overall grazing rate on labeled prey ( $\left.G_{\text {label }}\right)$ is calculated as:

$$
G_{\text {label }}=\lambda(1-z)
$$

where $G_{\text {label }}$ is estimated in units of ingested labeled prey per flagellate per base unit time (i.e. $1 \mathrm{~min}$ ). Assuming grazers do not discriminate between labeled and unlabeled prey, bacteria ingested per flagellate per unit time can be calculated by multiplying $G_{\text {label }}$ by the ratio of total bacteria to labeled prey.

\section{RESULTS}

\section{Rhynchomonas nasuta: distribution of ingested FLB}

Frequency distributions were collected by microscopy for a total of 32 Rhynchomonas nasuta samples incubated on 4 dates. Sample sizes ranged from 202 to 1592 flagellates, but were typically 300 to 400 , with means of 0.1 to 0.5 FLB ingested per flagellate. Sample sizes truncated at the one class were usually less than 50. Table 1 presents the observed frequency of the number of flagellates with $0,1, \ldots, 4$ FLB $\left(f_{x}\right)$ in a sample of 1592 flagellates. This table also includes expected frequencies $\left(F_{x}\right)$, log-likelihood ratios, and $\chi^{2}$ test statistics for each of the hypothesized distributions Test statistics exceeded critical values for the binomial and the Poisson, suggesting these were not appropriate models. However, both the truncated Poisson and the negative binomial models acceptably approximated this sample distribution. Acceptable fit of the sample distribution in Table 1 to the Poisson EZ model suggests the presence of 2 subpopulations, feeders, and non-feeders, the latter of which comprise $30 \%$ of the total population (the extra zeros). In contrast, fit of the sample to the negative binomial suggests subpopulations with a range of grazing rates that may extend to

Table 1. Observed frequencies of labeled bacteria in Rhynchomonas nasuta, expected frequencies $\left(F_{x}\right)$, and log-likelihood ratios $\left[f_{x} \ln \left(f_{x} / F_{x}\right)\right]$. (P-values must be greater than 0.05 for acceptance). NB = negative binomial, df $=$ degrees of freedom, na $=$ not applicable, $\mathrm{P}=$ probability of the observed value of $\chi^{2}$ or greater

\begin{tabular}{|c|c|c|c|c|c|c|c|c|c|c|}
\hline \multirow[t]{2}{*}{$x$} & \multirow{2}{*}{$f_{\mathrm{x}}$} & \multicolumn{4}{|c|}{ Expected frequencies $\left(F_{x}\right)$} & & \multicolumn{4}{|c|}{ Log-likelihood ratio } \\
\hline & & Poisson ${ }^{a}$ & Poisson $\mathrm{EZ}^{\mathrm{b}}$ & Binom ${ }^{c}$ & $\mathrm{NB}^{\mathrm{d}}$ & & Poisson & Poisson EZ & Binom. & NB \\
\hline 0 & 1163 & 1132.6 & 683.0 & 1115.3 & 1162.5 & & 30.804 & na & 48.706 & 0.500 \\
\hline 1 & 337 & 385.6 & 332.9 & 415.1 & 338.3 & & -45.400 & 4.125 & -70.243 & -1.297 \\
\hline 2 & 74 & 65.6 & 81.1 & 57.9 & 73.8 & & 8.916 & -6.780 & 18.156 & 0.200 \\
\hline 3 & 15 & 7.4 & 13.2 & 37 & 14.3 & & 10.599 & 1.916 & 2848 & 0.717 \\
\hline 4 & 3 & 0.7 & 1.8 & 3.7 & 3.1 & & 4.366 & 1.533 & 20.40 & -0.098 \\
\hline \multirow[t]{4}{*}{$\Sigma$} & 1592 & 1592 & 1112 & 1592 & 1592 & & 9.285 & 0.795 & 25.095 & 0.0214 \\
\hline & & & & & & $\chi^{2}=$ & 18.570 & 1.591 & 50.190 & 0.0427 \\
\hline & & & & & & $\mathrm{df}=$ & 3 & 2 & 3 & 2 \\
\hline & & & & & & $\mathrm{P}=$ & 0.0003 & 0.4514 & 0.0000 & 0.9789 \\
\hline
\end{tabular}


Table 2. Frequency distribution of test statistic percentiles for 32 samples of Rhynchomonas nasuta, expected frequencies $\left(F_{x}\right)$, and log-likelihood ratios $\left[f_{\mathrm{x}} \ln \left(f_{\mathrm{x}} / F_{\mathrm{x}}\right)\right]$. (p-values must be greater than 0.05 for acceptance $) . \mathrm{NB}=$ negative binomial, df $=$ degrees of freedom, $P=$ probability of the observed value of $\chi^{2}$ or greater

\begin{tabular}{|c|c|c|c|c|c|c|}
\hline \multirow{2}{*}{$\begin{array}{l}\text { Test statistic } \\
\text { percentile range }\end{array}$} & \multicolumn{2}{|c|}{ Frequency of test statistic percentile } & \multirow{2}{*}{$\begin{array}{l}\text { Expected } \\
\text { frequency }\end{array}$} & & \multicolumn{2}{|c|}{ Log-likelihood ratio } \\
\hline & Poisson EZ & NB & & & Poisson EZ & NB \\
\hline $0-10$ & 6 & 4 & 3.2 & & -1.1631 & -0.9400 \\
\hline $10-25$ & 5 & 4 & 4.8 & & -0.7293 & -1.4100 \\
\hline $25-50$ & 9 & 11 & 8.0 & & -0.9347 & 0.0000 \\
\hline $50-75$ & 7 & 8 & 8.0 & & 1.0600 & 3.5030 \\
\hline $75-90$ & 4 & 3 & 4.8 & & 0.2041 & -0.7293 \\
\hline $90-100$ & 1 & 2 & 3.2 & & 3.7716 & 0.8926 \\
\hline \multirow[t]{4}{*}{$\Sigma$} & 32 & 32 & 32.0 & & 2.2086 & 2.6326 \\
\hline & & & & $\chi^{2}=$ & 4.4173 & 2.6325 \\
\hline & & & & $\tilde{\mathrm{d} f}=$ & 5 & 5 \\
\hline & & & & $P=$ & 0.4910 & 0.7564 \\
\hline
\end{tabular}

zero. Table 2 shows a frequency distribution of the test statistic percentiles for the $32 R$. nasuta samples analyzed. Based on the 32 tested sample distributions of FLB in $R$. nasuta, both the Poisson EZ and the negative binomial models acceptably describe the distribution of ingested prey.

\section{Paraphysomonas vestita: distribution of ingested FLB and cytometric and microscopic grazing rates}

Fig. 5 displays $\mathrm{P}_{0(t)}$ data points for 3 replicate samples and 2 theoretical curves (Eq. 7). The first data $\mathrm{P}_{0(t)}$ point is estimated to be at $0.5 \mathrm{~min}$ rather than zero min to allow for time to mix FLB with the grazers and collect the aliquots prior to actual grazing cessation. Collection and grazing cessation of later aliquots could be timed more precisely. Good visual fit of the data points to the

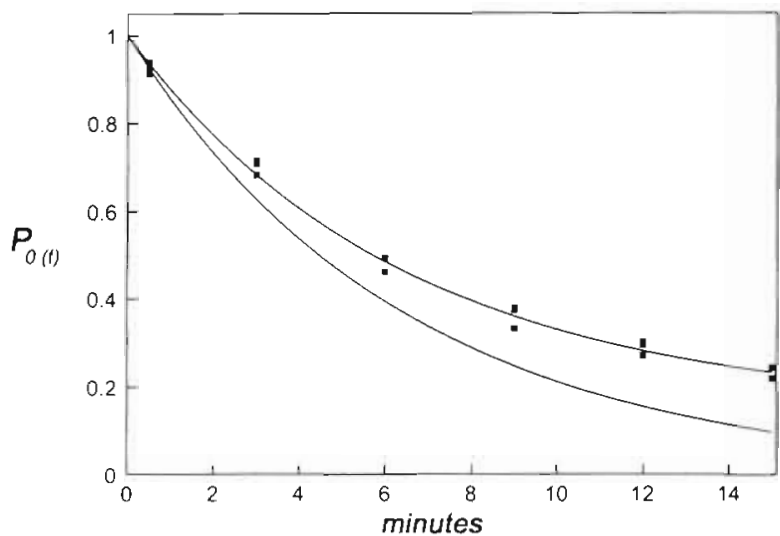

Fig. 5. Probability of zero $\left[\mathrm{P}_{0(t)}\right]$ versus time for Paraphysomonas vestita incubated with FLB, and Poisson EZ proba. bility of zero curves with the Poisson parameter $(\lambda)=0.15$ Data points ( 3 per time point) are solid squares; for the lower curve, $z=0$; for the upper curve, $z=0.14$ displayed Poisson EZ curve suggests that the sample approximates a Poisson with $\lambda=0.15$, and about $14 \%$ extra zeros $(z=0.14)$. On some sample dates, data $P_{0(t)}$ curves were parallel to theoretical curves, but slightly lower by a constant amount at all time points. This was thought to be due to the association of uningested FLB with the cluster of bacteria sometimes loosely attached to flagellates. In these cases, $P_{0(t)}$ points were overlaid on theoretical lines by the addition of a small sampledependent constant to data $\mathrm{P}_{0\langle t\rangle}$.

Table 3 shows microbial abundances, accepted model distributions, and grazing rate estimates for 4 experiments based on microscopic and cytometric analysis of ingested FLB. Microscopic frequency distributions included 100 to 415 flagellates for each sample, with a minimum of 95 ingested FLB. Grazing rates in Table 3 are single sample estimates, with the exception of Expt $\mathrm{C}$, which is the mean of triplicate samples. The standard deviations for Expt $C$ grazing rates were 0.07 and 0.05 for microscopic and cytometric analyses, respectively. The sample distribution of ingested FLB in Expt $D$ did not fit any of the tested distributions; however, parameter estimations were exceptionally poor due to significant truncation of the right tail. This was a consequence of a relatively high concentration of FLB in conjunction with a rapid grazing rate that yielded a microscopy-determined mean of 3.5 FLB flagellate $^{-1}$ after $12 \mathrm{~min}$ incubation. Given the maximum of 6 ingested FLB that could be confidently enumerated under these viewing conditions, this sample had a relatively large proportion of grazers that ingested more than 6 FLB. With the calculation methods applied, excessive right truncation would result in underestimation of microscopically determined grazing rates in Expt D. All other sample distributions assessed by microscopy acceptably fit negative binomial and truncated Poisson distributions (used to test a Poisson EZ), and 2 out of 3 also fit a Poisson. 
Table 3. Paraphysomonas vestita and prey abundances, distributions of ingested FLB based on microscopy frequency distributions, and grazing rates based on microscopy frequency distributions and cytometry probability of zero $\left[\mathrm{P}_{0(\ell)}\right]$ curves. $(\mathrm{NB}=\mathrm{negative}$ binomial, $\mathrm{TP}=$ truncated Poisson, $\mathrm{P}=$ Poisson)

\begin{tabular}{|lcccccc|}
\hline Sample & Grazers $\mathrm{ml}^{-1}\left(\times 10^{4}\right)$ & Prey $\mathrm{m}^{-1}\left(\times 10^{7}\right)$ & $\%$ FLB & $\begin{array}{c}\text { Accepted distribution } \\
(\alpha=0.05)\end{array}$ & $\begin{array}{c}\text { Bacteria grazer }^{-1} \mathrm{~min}^{-1} \\
\text { Via cytometry }\end{array}$ \\
\hline A & 2.2 & 5.0 & 10.8 & NB, TP, P & 0.9 & 1.0 \\
B & 1.7 & 4.9 & 12.7 & NB, TP & 0.3 & 0.3 \\
C & 3.9 & 6.7 & 10.9 & NB, TP, P & 0.7 & 1.2 \\
D & 40.5 & 2.9 & 22.1 & Poor test & 1.3 \\
\hline
\end{tabular}

Table 4 shows microbial abundances and grazing rate estimates based on uptake of fluorescent microspheres. Two cytometrically determined grazing rates are estimated for each sample; 1 rate is based on $\mathrm{P}_{0(t)}$ curves, and the second rate is based on microsphere frequency distributions (unlike FLB, microsphere frequency distributions could be quantified by flow cytometry). Grazing rates in Table 4 are single sample estimates, with the exception of Expt F, which is the mean of triplicate samples. The standard deviations for Expt $F$ were 0.089 and 0.010 for the $P_{0(t)}$ curve and frequency distribution methods, respectively. None of the microsphere frequency distributions (with 40000 flow cytometrically analyzed flagellates) acceptably fit a Poisson or a Poisson EZ. However, $P_{0(t)}$ curve grazing rate estimates of the microsphere samples (based on the assumption of a Poisson EZ) were similar to the rate estimates based on frequency distributions. All microsphere frequency distributions acceptably fit negative binomial distributions for aliquots removed after 3 and 6 min incubation with labeled prey, but acceptable fit was not always found in longer incubations. As incubation time increased, the right tails of sample distributions became better defined, making deviation from the negative binomial model easier to detect.

\section{DISCUSSION}

Both the Poisson EZ and the negative binomial were found to be generally acceptable models of the distribution of ingested prey in cultures of a surface feeding flagellate with a cytostome (Rhynchomonas nasuta) and a phagocytic grazer of free-swimming bacteria (Paraphysomonas vestita). While both of these models suggest the presence of subgroups of flagellates with different grazing rates, they have contrasting implications with regard to the number of flagellate subgroups. Acceptance of the Poisson EZ model suggests 2 categories of grazers: a non-feeding group, and a feeding group with a single grazing rate. In contrast, the negative binomial suggests a series of different grazing rates that may extend to zero. Acceptance of 2 models with different biological implications precludes acceptance of the specific theoretical implications of either of these models without further tests. Much larger sample sizes are often necessary for accurate assessment of the characteristics of low mean distributions. In this study, cytometric analysis of microspheres in flagellates included at least 40000 flagellates per sample. The distribution of microsphere uptake in these large samples could not be acceptably fit to either a Poisson or a Poisson EZ. Even the more robust negative binomial model no longer provided acceptable fit when the right tail of the distribution was allowed to further develop with longer incubation times. Loss of acceptance of these relatively simplistic models supports the findings of Hatzis et al. (1990, 1993b) that suggest the use of more complex models to describe the distribution of fluorescent microspheres in the ciliate Tetrahymena. When Tetrahymena was offered fluorescent microspheres as the only available prey, individual cell grazing was described by a Poisson (Gerritsen et al. 1987, Hatzis et al. 1990, 1993b),

Table 4. Paraphysomonas vestita and prey abundances, distribution of ingested microspheres based on cytometry histograms, and grazing rates based on cytometry frequency distributions and cytometry probability of zero $\left[\mathrm{P}_{0(t)}\right]$ curves. $(\mathrm{NB}=\mathrm{negative}$ binomial).

\begin{tabular}{|c|c|c|c|c|c|c|}
\hline \multirow[t]{2}{*}{ Sample } & \multirow[t]{2}{*}{ Grazers $\mathrm{ml}^{-1}\left(\times 10^{4}\right)$} & \multirow[t]{2}{*}{ Prey $\mathrm{ml}^{-1}\left(\times 10^{7}\right)$} & \multirow{2}{*}{$\begin{array}{c}\% \\
\text { spheres }\end{array}$} & \multirow{2}{*}{$\begin{array}{l}\text { Accepted distribution } \\
\qquad(\alpha=0.05)\end{array}$} & \multicolumn{2}{|c|}{ Bacteria grazer ${ }^{-1} \min ^{-1}$} \\
\hline & & & & & Frequency distribution & $\mathrm{P}_{0(\eta)^{\circ}}$ curve \\
\hline E & 13.3 & 4.2 & 10.0 & NB & 1.0 & 0.8 \\
\hline $\mathrm{F}$ & 1.3 & 3.2 & 5.6 & NB & 0.4 & 0.4 \\
\hline G & 5.7 & 3.6 & 8.9 & NB & 0.3 & 0.4 \\
\hline
\end{tabular}


while populations with individual variability were described by compound Poisson distributions that are more complex than the negative binomial (Hatzis et al. 1990, 1993b).

However, with recognition that the theoretical implications of the simple Poisson distribution used by Gonzalez (1999) and the Poisson EZ used for the cytometry method of this study may not be correct, rough fit of samples to these distributions may allow use of faster methods for estimating bacterivory. The acceptability of these distributions may be greatest when sample means and sample sizes are relatively low, particularly for the simplest case of the Poisson. The results of this study suggest that mean grazing rates estimated from $\mathrm{P}_{0(t)}$ curves of the Poisson EZ model are often similar to rates estimated with fluorescence microscopy. While better estimates of grazing rate variance are needed to determine the relative bias of each method, the many potential sources of unquantified measurement error preclude statements on which method is more representative of actual grazing rates. In this study, potential measurement error factors (in addition to representativeness of FLB prey) include counting error intrinsic to each analysis method, different effects of $\mathrm{NiCl}_{2}$ and low temperature fixation on FLB retention, and sample deviation from the Poisson EZ model. The significance of deviation from the Poisson EZ model can be examined by comparison of grazing rates on microspheres estimated with identical samples by both frequency distribution and $\mathrm{P}_{0(t)}$ curve methods. Under these conditions, the primary difference in the average rate estimates is due to deviation from the Poisson EZ model. Greater similarity between the cytometric estimates of microsphere uptake (Table 4) than between the cytometric and microscopic estimates of FLB uptake (Table 3) suggests that error due to deviation from the Poisson EZ model was relatively small.

When using the $\mathrm{P}_{0(t)}$ curve method for estimation of flagellate grazing rates on FLB, cytometric overlap of grazer and prey populations will likely vary with different populations. Natural samples containing a wide range of grazer species may have relatively greater overlap of grazer and prey populations. However, modest but constant error due to a small fraction of free FLB in the grazer window, or due to error in cytometric windows designated for distinguishing grazers with and without ingested labeled prey, should not significantly affect grazing rate estimates because this method depends on change in $\mathrm{P}_{0(\ell)}$ rather than on accurate measurement of $\mathrm{P}_{0(\imath)}$ at a given time. Careful assessment of good visual fit of the sample $\mathrm{P}_{0(t)}$ curve to the best theoretical $P_{0(1)}$ curve is important, particularly when new sample types or conditions are being tested. It is possible that acceptable fit to the theoretical $P_{0(t)}$ curve will be lost in samples with a wider dispersion of grazing rates. This may occur in samples that have been exposed to sub-lethal stress (Bratvold 1995), as well as in natural community samples with multiple grazer species. Poor sample fit to the Poisson EZ model may be indicated by inconsistent deviation of data points from the theoretical curve for a single sample, but a consistent pattern of deviation among replicate samples. Microscopical assessment of samples with poor fit to the best theoretical curve would be necessary to validate applicability of this cytometry method for these samples. When a Poisson EZ $\mathrm{P}_{0(t)}$ curve provides good fit to observed $\mathrm{P}_{0(t)}$ curves, the cytometry method may lose precision when labeled prey concentrations are selected such that the mean number of labeled prey per flagellate per minute is too high (e.g. $>0.6$ for $15 \mathrm{~min}$ incubations). Conversely, when the mean number of labeled prey per grazer is too low (e.g. <0.05 for $15 \mathrm{~min}$ incubation times), the fraction of extra zeros in the population cannot be confidently estimated. Thus, proper selection of FLB concentrations is essential for this method. Paired incubations could be conducted with 1 of the pairs containing higher concentrations of FLB to increase the chances of obtaining an estimate of $z$ before digestion time is reached. However, the validity of this approach is uncertain until it is determined if $z$ remains constant within the range of FLB additions applied. Changes or constancy in $z$ with different FLB concentrations may provide indirect evidence on the validity of the theoretical implication of non-feeders.

Cytometric studies of Tetrahymena grazing on fluorescent microspheres suggest that a proportion of the ciliate population are non-feeders. This proportion was estimated to be 20 to $30 \%$ of exponential phase cultures, and nearly $100 \%$ of starved cultures (Hatzis 1993a). Applying the Poisson EZ model, extra zeros in the late exponential to early stationary phase Rhynchomonas nasuta cultures of this study were estimated to range from 30 to $80 \%$. Extra zeros in mid- to lateexponential phase Paraphysomonas vestita cultures ranged from 5 to $46 \%$. While these ranges appear to be roughly consistent with the non-feeder ranges found by Hatzis et al. (1993a), the extra zeros of this study may represent both true non-feeders and the increased probability of subpopulations with lower grazing rates not ingesting any labeled prey during the incubation time. The findings in this study of acceptable fit of exponential phase $P$. vestita cultures to a simple Poisson may have been enabled by a relatively small fraction of non-feeding cells in these samples in conjunction with the relatively small sample sizes used for microscopy analysis (i.e. several hundred grazers).

When recognizing model suggestions of a non-feeding subpopulation, some possible explanations for non-feeders warrant consideration. It has been suggested that glutaraldehyde fixation causes some fla- 
gellates to egest food vacuole contents (Sieracki et al. 1987). If it is assumed that flagellates containing 1 labeled bacterium are as likely to egest food vacuole contents as flagellates containing 2, 3, and 4 labeled bacteria, egestion upon fixation would lower the mean of the ingested bacteria per flagellate distribution, but would not change the distribution type. Thus, egestion of food vacuole contents upon fixation cannot explain apparent non-feeders. It is possible that some nonfeeders may occur in the surface feeding Rhynchomonas nasuta population if some flagellates remained in the water column, unassociated with surfaces during the stagnant $15 \mathrm{~min}$ incubation and were unable to feed while suspended. However, this possibility seems unlikely to be able to fully account for the variable and sometimes very high proportion of observed extra zeros. In the $R$. nasuta experiments, grazing incubations were conducted when flagellates were in lateexponential or early-stationary phase, when only a small proportion of the population is unable to feed as a result of division and mouth-part formation. Thus, the extra zero estimates ranging from 30 to $80 \%$ of the total population in the samples of Table 2 may suggest another life cycle stage during which feeding is reduced or does not occur. Two general behaviors of $R$. nasuta were observed in these cultures: (1) gliding, scouring, and feeding along surfaces, and (2) rapidly twirling through the water column, which appeared to be more common in older cultures. This later behavior may be similar to the 'swarmers' described by Fenchel (1982) at the onset of starvation. If individuals remain engaged in only 1 of these behavior types throughout a grazing incubation, the extra zeros in the Poisson EZ model may correlate with the number of swarmers; but extra zeros would not be equivalent to swarmers if the Poisson EZ is a simplification of a more complex compound Poisson distribution.

Overall, studies conducted with alternative methods are needed to determine if there is a correlation between extra zeros of the Poisson EZ model and a nonfeeding subpopulation of grazers, or if the Poisson EZ is merely a mathematical description of prey uptake when uptake is a low probability event; analyses of grazing on microspheres suggests the latter. However, the fit of the Poisson EZ and negative binomial to sample distributions of FLB in 2 nanoflagellate types with very different feeding mechanisms and different growth phases suggests that these models may provide simplified, robust descriptions of the heterogeneous distribution of FLB in flagellate grazers. The fit of samples to the Poisson EZ allows grazing rates to be estimated from the change in the fraction of cells without FLB $\left[\mathrm{P}_{\mathrm{O}(t)}\right]$ using flow cytometry. While the cytometry method presented can provide rapid estimates of cultured flagellate grazing rates, making it easier to test the effects of multiple variables on grazing, additional studies are needed to determine the applicability of these methods for analysis of natural communities.

Acknowledgements. We are grateful for the cytometry assistance of B. Dien, computer software and hardware assistance of R. E. Circle, and flagellate identifications by T. A. Nerad, E. L. Lim and D. A. Caron. We thank A. H. El-Shaarawi, C. J Sutton, G. B. McManus, and 2 anonymous reviewers for their helpful comments. This study was supported in part by a George Mason University Graduate Student Fellowship

\section{LITERATURE CITED}

Andersson A, Larsson U, Hagström $\AA$ (1986) Size-selective grazing by a microflagellate on pelagic bacteria. Mar Ecol Prog Ser 33:51-57

Bishop YMM, Fienberg SE, Holland PW (1975) Discrete multivariate analysis: theory and practice. The MIT Press Cambridge, MA, p 311-332

Bjørnsen PK, Riemann B, Horsted SJ, Nielsen TG, Pock-Sten $J$ (1988) Trophic interactions between heterotrophic nanoflagellates and bacterioplankton in manipulated seawater enclosures. Limnol Oceanogr 33:409-420

Bliss CI, Fisher RA (1953) Fitting the negative binomial to biological data. Biometrics 9:177-200

Bloem J, Albert C, Bär-Gillissen MJB, Berman T, Cappenberg IE (1989) Nutrient cycling through phytoplankton, bacteria and protozoa, in selectively filtered Lake Vechten water J Plankton Res 11:119-131

Bratvold D (1995) Methodological development and distribution analysis of flagellate grazing on fluorescently labeled bacteria. PhD dissertation, George Mason University, Fairfax, VA

Caron DA, Goldman JC (1988) Dynamics of protistan carbon and nutrient cycling. J Protozool 35:247-249

Davis PG, Sieburth JMcN (1984) Estuarine and oceanic microflagellate predation of actively growing bacteria: estimation by frequency of dividing-divided bacteria. Mar Ecol Prog Ser 19:237-246

El-Shaarawi AH, Esterby SR, Dutka BJ (1981) Bacterial density in water determined by Poisson or negative binomial. Appl Environ Microbiol 41:107-116

Fenchel T (1982) Ecology of heterotrophic microflagellates, III. Adaptations to heterogeneous environments. Mar Ecol Prog Ser 9:25-33

Fenchel T (1987) Ecology of the protozoa: the biology of freeliving phagotrophic protists. Springer-Verlag, New York

Ferrier C, Rassoulzadegan F (1991) Density-dependent effects of protozoans on specific growth rates in pico- and nanoplankton assemblages. Limnol Oceanogr 36:657-669

Ferrier-Pagès C, Rassoulzadegan F (1994) Seasonal impact of the microzooplankton on pico- and nanoplankton growth rates in the northwest Mediterranean Sea. Mar Ecol Prog Ser 108:283-294

Fredrickson AG, Hatzis C, Srienc F (1992) A statistical analysis of flow cytometric determination of phagocytosis rates. Cytometry 13:423-431

Gerritsen J, Sanders RW, Bradley SW, Porter KG (1987) Individual feeding variability of protozoan and crustacean zooplankton analyzed with flow cytometry. Limnol Oceanogr 32:691-699

Goldman JC, Caron DA (1985) Experimental studies on an omnivorous microflagellate: implications for grazing and 
nutrient regeneration in the marine microbial food chain. Deep-Sea Res 32:899-915

Goldman JC, Caron DA, Andersen DK, Dennett MR (1985) Nutrient cycling in a microflagellate food chain: I. Nitrogen dynamics. Mar Ecol Prog Ser 24:231-242

Gonzalez JM (1999) Bacterivory rate estimates and fraction of active bacterivores in natural protist assemblages from aquatic systems. Appl Environ Microbiol 65:1463-1469

Gonzalez JM, Iriberri J, Egea L, Barcina I (1990) Differential rates of digestion of bacteria by freshwater and marine phagotrophic protozoa. Appl Environ Microbiol 56: $1851-1857$

Gumbel EJ (1968) Ladislaus von Bortkiewicz. An international encyclopedia of social sciences, Vol 2. Macmillan, New York, p 128-131

Hatzis C, Sweeney PL, Srienc F, Fredrickson AG (1990) A discrete stochastic model for microbial filter feeding: a model for feeding of ciliated protists on spatially uniform. nondepletable suspensions. Math Biosci 102:127-181

Hatzis C, Srienc F, Fredrickson AG (1993a) Feeding heterogeneity in ciliate populations: effects of culture age and nutritional state. Biotechnol Bioeng 43:371-380

Hatzis C, Sweeney PJ, Srienc F, Fredrickson AG (1993b) Determination of cellular rate distributions in microbial cell populations: feeding rates of ciliated protozoa. Biotechnol Bioeng 42:284-294

Hollisbaugh JT, Fuhrman JA, Azam F (1980) Radioactively labeling of natural assemblies of bacterioplankton for use in trophic studies. Limnol Oceanogr 25:172-181

Hondeveld BJM, Bak RPM, van Duyl FC (1987) Bacterivory by heterotrophic nanoflagellates in marine sediment measured by fluorescently labeled bacteria. Appl Environ Microbiol 53:958-965

Hutchins DA, Bruland KW (1994) Grazer-mediated regeneration and assimilation of $\mathrm{Fe}, \mathrm{Zn}$, and $\mathrm{Mn}$ from planktonic prey. Mar Ecol Prog Ser 110:259-269

Landry MR, Lehner-Fournier JM, Sundstrom JA, Fagerness VL, Selph KE (1991) Discrimination between living and heat-killed prey by a marine zooflagellate, Paraphysomonas vestita (Stokes). J Exp Mar Biol Ecol 146:139-151

Lavin DP, Fredrickson AG, Scrienc F (1990) Flow cytometric measurements of rates of particle uptake from dilute suspensions by a ciliated protozoan. Cytometry 11:875-882

Linley EAS, Newell RC, Lucas MI (1983) Quantitative relationships between phytoplankton, bacteria, and heterotrophic microplankton in shelf waters. Mar Ecol Prog Ser 12:77-89

McManus GB, Fuhrman JA (1986) Bacterivory in seawater studied with the use of inert fluorescent particles. Limnol Oceanogr 31:420-426

McManus GB, Okubo A (1991) On the use of surrogate food particles to measure protistan ingestion. Limnol Oceanogr 36:613-617

Nerad TA, Daggett PM (1992) Cultivation of selected colorless crypromonads. In: Soldo AT (ed) Protocols of protozoology.

Editorial responsibility: Robert Sanders

Philadelphia, Pennsylvania, USA
Society of Protozoologists, Lawrence, KS, p A7.1-A7.3

Nygaard K, Hessen DO (1990) Use of ${ }^{14} \mathrm{C}$-protein-labeled bacteria for estimating clearance rates by heterotrophic and mixotrophic flagellates. Mar Ecol Prog Ser 68:7-14

Nygaard K, Børsheim KY, Thingstad TF (1988) Grazing rates on bacteria by marine heterotrophic microflagellates compared to uptake rates of bacteria-sized monodispersed fluorescent latex beads. Mar Ecol Prog Ser 44:159-165

Pace ML, Bailiff MD (1987) Evaluation of a fluorescent microsphere technique for measuring the grazing rates of phagotrophic microorganisms. Mar Ecol Prog Ser 40 $185-189$

Porter KG, Feig SY (1980) The use of DAPI for identifying and counting aquatic microflora. Limnol Oceanogr 25:942-946

Roman MR, Ducklow HW, Fuhrman JA, Garside C, Gilbert PM, Malone TC, McManus GB (1988) Production, consumption and nutrient cycling in a laboratory mesocosm Mar Ecol Prog Ser 42:39-52

Rothhaupt KO (1992) Stimulation of phosphorous-limited phytoplankton by bacterivorous flagellates in laboratory experiments. Limnol Oceanogr 36:750-759

Selmer JS, Ferrier-Pagès $C$, Cellario $C$, Rassoulzadegan $F$ (1993) New and regenerated production in relation to the microbial loop in the NW Mediterranean Sea. Mar Ecol Prog Ser 100:71-83

Sherr BF, Sherr EB, Berman T (1983) Grazing, growth, and ammonium excretion rates of heterotrophic microflagellates fed four species of bacteria. Appl Environ Microbiol 45:1196-1201

Sherr BF, Sherr EB, Fallon RD (1987) Use of monodispersed, fluorescently labeled bacteria to estimate in situ protozoan bacterivory. Appl Environ Microbiol 53:958-965

Sherr BF, Sherr EB, Hopkinson CS (1988a) Trophic interactions with pelagic microbial communities: indications of feedback regulation of carbon flow. Hydrobiologia 159 $19-26$

Sherr BF, Sherr EB, Rassoulzadegan F (1988b) Rates of digestion of bacteria by marine phagotrophic protozoa: temperature dependence. Appl Environ Microbiol 54:1091-1095

Sherr BF, Sherr EB, Pedrós-Alió C (1989) Simultaneous measurement of bacterioplankton production and protozoan bacterivory in estuarine water. Mar Ecol Prog Ser 54: 209-219

Sieburth JMCN, Davis PG (1982) The role of heterotrophic nanoplankton in the grazing and nurturing of planktonic bacteria in the Sargasso and Caribbean Seas. Ann Inst Oceanogr Paris 58(S):285-296

Sieracki ME, Haas LW, Caron DA, Lessard EJ (1987) Effect of particle retention by microflagellates: underestimation of grazing rates. Mar Ecol Prog Ser 38:251-258

Sokal RR, RohIf FJ (1981) Biometry, 2nd edn. WH Freeman and $\mathrm{Co}$, New York

Stout J (1977) The role of protozoa in nutrient cycling and energy flow. In: Alexander $M$ (ed) Advances in microbial ecology, Vol 4. Plenum Press, New York, p 1-50

Submitted: March 28, 1999; Accepted: September 9, 1999

Proofs received from author(s): February 3, 2000 